\title{
The cathelicidins LL-37 and rCRAMP are associated with pathogenic events of arthritis in humans and rats
}

\author{
Markus H Hoffmann, ${ }^{1}$ Heiko Bruns, ${ }^{2}$ Liselotte Bäckdahl, ${ }^{1}$ Petra Neregård, ${ }^{3}$ \\ Birgit Niederreiter, ${ }^{4}$ Martin Herrmann, ${ }^{5}$ Anca Irinel Catrina, ${ }^{3}$ Birgitta Agerberth, ${ }^{6}$ \\ Rikard Holmdahl ${ }^{1}$
}

- Additional data are published online only. To view these files please visit the journal online (http://dx.doi.org/ 10.1136/annrheumdis-2012202218)

${ }^{1}$ Division of Medical Inflammation Research, Department of Medical Biochemistry and Biophysics, Karolinska Institutet,

Stockholm, Sweden

${ }^{2}$ Department of Internal

Medicine 5, University of

Erlangen-Nuremberg, Erlangen, Germany

${ }^{3}$ Rheumatology Unit, Department of Medicine at the Karolinska University Hospital Solna, Sweden

${ }^{4}$ Division of Rheumatology, Department of Internal

Medicine 3, Medical University of Vienna, Vienna, Austria ${ }^{5}$ Department of Internal Medicine 3, University of Erlangen-Nuremberg, Erlangen, Germany

${ }^{6}$ Division of Chemistry II, Department of Medical Biochemistry and Biophysics, Karolinska Institutet, Stockholm, Sweden

\section{Correspondence to} Dr Markus H Hoffmann, Department of Internal Medicine 3, University of Erlangen-Nuremberg, Glückstrasse 4a, 91054 Erlangen, Germany; markus. hoffmann@uk-erlangen.de

$\mathrm{BA}$ and $\mathrm{RH}$ contributed equally.

Accepted 21 October 2012 Published Online First 21 November 2012

\section{ABSTRACT}

Background In rheumatoid arthritis (RA), neutrophil granulocytes fuel inflammation and damage tissue in the joint by releasing cytotoxic agents, antimicrobial peptides, proteases and other inflammatory mediators. The human cathelicidin LL-37 has recently been implicated in the development of systemic lupus erythematosus and psoriasis.

Objective To elucidate if antimicrobial peptides (AMPs) contribute to the pathogenesis of arthritis.

Methods Expression of LL-37 was determined in synovial membranes from patients with arthritis and control subjects. Expression of the rat cathelicidin rCRAMP and defensins was characterised in joints, blood and secondary lymphoid organs during pristane-induced arthritis (PIA) in rats and in a transfer model of PIA induced by CD4 T cells. Serum samples of rats with arthritis were tested for $\lg G$ and $\lg M$ autoantibodies against rCRAMP by immunoblot and for interferon (IFNo) by ELISA.

Results Cathelicidins are strongly upregulated in RA synovial membranes and in joints from rats with arthritis as compared with healthy joints. Expression was most prominent in neutrophil granulocytes and macrophages/ osteoclasts. Cathelicidin expression is also upregulated in the blood and spleen of pristane-injected rats, with strongest expression detected in activated CD62L- cells coexpressing granulocyte and monocyte markers. Pristane injection caused accumulation of low-density granulocytes in the blood. After pristane injection, the increased expression of rCRAMP coincided with higher levels of cell death, raised levels of interferon (IFN) $\alpha$ and development of autoantibodies.

Conclusions Our results show strong upregulation of cathelicidins and $\beta$-defensins coinciding with pathological events of arthritis. Higher expression and release of AMPs might contribute to development and/or maintenance of disease by systemic or local mechanisms.

\section{INTRODUCTION}

Anti-microbial peptides (AMPs) such as the cathelicidin LL-37 and defensins have recently been implicated in the pathogenesis of autoimmune diseases. ${ }^{1-4}$ In addition to their antimicrobial activities, AMPs possess potent immunomodulatory properties. ${ }^{5}$ LL-37 attracts neutrophils, monocytes, $\mathrm{T}$ cells and mast cells ${ }^{6}$ and induces a wide range of genes involved in cell adhesion, communication and motility. ${ }^{7}$ In psoriasis, LL-37 produced by keratinocytes and neutrophils appears to contribute to the pathogenesis by inducing production of type I interferons (IFNs) by altering membrane fluidity ${ }^{8}$ or via the formation of complexes with locally released self-DNA and self-RNA, thereby leading to extended activation of Toll-like receptor (TLR)-7 and TLR-9. ${ }^{1-4}$ In the serum samples of patients with systemic lupus erythematosus (SLE), immune complexes of AMPs and self-DNA produced by activated neutrophils in the form of neutrophil extracellular traps trigger activation of TLR9. Furthermore, patients with SLE were found to develop autoantibodies to both self-DNA and AMPs. $^{2} 3$

Patients with $\operatorname{SLE}^{9} 10$ and a subgroup of patients with rheumatoid arthritis (RA) ${ }^{11}$ display a type I IFN signature of their peripheral blood mononuclear cell (PBMC) gene expression. In view of the importance of AMPs for the development of SLE and psoriasis they may possibly also stimulate TLR pathways involved in other autoimmune diseases characterised by reactivity to nucleic acids, such as arthritis.

Under inflammatory conditions, such as in psoriasis or SLE ${ }^{12} 13$ and in the joints of patients with $\mathrm{RA},{ }^{14}$ AMPs are induced at the transcriptional level. Interestingly, also, expression of proteinase 3, which mediates LL-37 processing and release from neutrophil granulocytes, is increased in patients with RA. ${ }^{15}$ Therefore, we aimed to investigate the expression of AMPs during the course of RA and experimental arthritis.

Arthritis in susceptible rat strains can be provoked by intradermal or subcutaneous injection of the hydrocarbon oil pristane (2,6,10,14-tetramethylpentadecane) ${ }^{16}$ Pristane-induced arthritis (PIA) shares many pathological features with RA, such as symmetrical involvement of peripheral joints, a chronic-relapsing disease course and the presence of rheumatoid factor. PIA also shares several features with SLE. Thus, nucleic acid-restimulated macrophages render $\mathrm{T}$ cells arthritogenic in the transfer model of PIA. Furthermore, reactivity against the nucleic acid-associated autoantigen hnRNP-A2 has been detected in PIA. ${ }^{17}$ PIA is therefore a well-suited model for analysing the role of AMPs in the development of arthritis. 


\section{MATERIALS AND METHODS}

Patients

Synovial samples were obtained by arthroscopy or open surgery from an area adjacent to the cartilage-pannus junction from 49 patients with early and longstanding RA and 10 healthy volunteers at the Karolinska University Hospital. Samples were snapfrozen in liquid nitrogen and maintained at $-70^{\circ} \mathrm{C}$ until sectioned. All experiments were approved by the local ethical committee.

\section{Experimental arthritis}

Dark Agouti (DA) rats originating from Harlan were bred and maintained under specific pathogen-free conditions (Felasa II) at the Division of Medical Inflammation Research, Karolinska Institute. Experiments were performed on rats frequencymatched for age and sex and evaluated blindly. PIA was induced by a subcutaneous injection of $150 \mu l$ pristane (Sigma-Aldrich, St Louis, USA) in the tail.

\section{Peptide/protein extraction}

Tissues (paws, spleens, lymph nodes, livers) were homogenised in $60 \%$ acetonitrile/ $1 \%$ trifluoroacetic acid. Extraction from blood was performed in pelleted whole blood (including erythrocytes) or from PBMCs/neutrophils isolated as previously described. ${ }^{18}$ Debris was removed by centrifugation and the supernatants were lyophilised and dissolved in $0.1 \%$ trifluoroacetic acid. Proteins and peptides were then enriched by reverse phase chromatography using Oasis cartridges (Waters, Milford, USA).

\section{Western blotting}

Denaturing sodium dodecyl sulphate-polyacrylamide gel electrophoresis was performed using $2-14 \%$ gradient gels (NuPAGE, Invitrogen). The peptide/protein material was then blotted to polyvinylidene difluoride membranes (Invitrogen). Membranes were blocked for $1 \mathrm{~h}$ with phosphate-buffered saline (PBS) $/ 5 \%$ milk powder and incubated for $1 \mathrm{~h}$ with a polyclonal affinity-purified IgG anti-rCRAMP antibody raised in rabbit (Innovagen, Lund, Sweden). After washing, the membranes were incubated for $1 \mathrm{~h}$ with peroxidase-conjugated antirabbit secondary antibody (GE Healthcare, Little Chalfont, UK). Finally, bands were visualised by enhanced chemiluminescence (GE Healthcare). Densitometric analysis was performed using ImageJ software (Rasband, WS, ImageJ, NIH, Bethesda, USA). Full-length synthetic rCRAMP (Innovagen) with the sequence

RFKKISRLAGLLRKGGEKFGEKLRKIGOKIKDFFOKLAPEIEQ$\mathrm{COOH}^{19}$ was used as positive control.

\section{Analysis of autoantibodies}

For detection of autoantibodies to rCRAMP, serum samples were taken from rats at days $0,6,14$ and 25 after pristane injection or during the chronic phase of PIA (day 100+) or at day 6 and 18 after cell transfer. Synthetic rCRAMP peptide was transferred to polyvinylidene difluoride membranes by western blot. Membranes were cut into strips, which were incubated with individual or pooled rat serum samples (diluted 1:50 in PBS/1\% milk powder) over night at $4^{\circ} \mathrm{C}$. As a control for specificity, some of the samples were preincubated with rCRAMP or $\beta$-defensin 1 (Innovagen) and some of the strips were preincubated with rabbit anti-rCRAMP or rabbit IgG from pooled rabbit serum (BioCare Medical). Detection was performed with a peroxidase-conjugated goat anti-rat IgG (Jackson Immunoresearch,
West Grove, USA) or anti-rat IgM, and bands were visualised by enhanced chemiluminescence (GE Healthcare).

Measurement of IFNo in serum

Serum/plasma levels of IFN $\alpha$ were detected in naïve rats and in rats $6,12,19$ and 21 days after pristane injection or after cell transfer, respectively, according to the instructions of the ELISA kit (Uscn Life Science, Wuhan, China).

\section{Flow cytometry}

Anti-rat granulocytes (His48)-fluorescein isothiocyanate (FITC), CD62L, anti-CD45RA (OX33)-phycoerythrin (PE), anti-CD45R (His24)-PE, anti-CD62L (HRL1)-biotin, anti-CD161a-biotin, streptavidin-FITC, streptavidin-PE were from BD Biosciences (Franklin Lakes, USA); anti-CD11b/c, anti-CD11c (8A2)-FITC, anti-CD68 (ED1)-Alexa Fluor 647, anti-CD68 (ED1)-FITC from Serotec (Oxford, UK); anti- $\gamma \delta$ T cell receptor (TCR) (V65)-PE from Biolegend (San Diego, USA). Antibodies to rCRAMP (Innovagen), rat $\beta$-defensin 1 (Santa Cruz Biotechnology, Santa Cruz, USA) and rat $\alpha$-defensin 4 (Santa Cruz) were labelled with Alexa Fluor 647 using the APEX labelling kit (Invitrogen).

Antibodies were applied to total blood, to isolated PBMCs/ neutrophils or to synovial fluid (SF) collected from rat ankle joints by lavage using cooled PBS and a 27G needle (BD Microlance). To obtain a sufficient number of cells, SF of three naive rats had to be pooled in some of the experiments. For intracellular staining (rCRAMP, defensins, CD68), cells were permeabilised and fixed with Cytofix/Cytoperm (BD Biosciences) before staining. In all experiments, control antibodies of the respective IgG isotypes were included. Flow cytometry was performed on a FACS Calibur machine (BD Biosciences) and was analysed using FloJo software.

\section{Immunohistochemistry and immunofluorescence microscopy}

For LL-37 expression analysis in humans, cryostat sections of synovial membranes were fixed for 20 min with $2 \%$ formaldehyde and dissolved in PBS at $4^{\circ} \mathrm{C}$. Synovial LL-37, CD3, CD31, $\mathrm{CD} 66 \mathrm{~b}$ and CD68 were detected using an affinity-purified rabbit polyclonal IgG anti-LL-37 (Innovagen), a mouse IgG1 antihuman CD66b (Beckman Coulter, Marseille, France), mouse IgG1 anti-human CD68, CD31 and CD3 (DakoCytomation, Glostrup, Denmark), respectively. Isotype controls using rabbit Ig and mouse IgG1 (both from DakoCytomation) were included. Visualisation was performed using diaminobenzidine, goat antirabbit IgG-Alexa Fluor 546 (Invitrogen) and goat anti-mouse IgG1-Alexa Fluor 488 (Invitrogen).

To assess the expression of rCRAMP by immunohistochemistry, serial paraffin sections of hind paws from naive and arthritic DA rats were deparaffinised and rehydrated. After blocking endogenous peroxidase using hydrogen peroxidase, sections were incubated for $60 \mathrm{~min}$ with rabbit anti-rCRAMP and for 30 min with a biotinylated horse anti-rabbit IgG antibody $(\mathrm{Ab})$, followed by incubation with the Vectastain Elite reagent (Vector Laboratories), leading to a brown staining of rCRAMP-expressing cells. Finally, slides were counterstained with haematoxylin (Merck). As a negative control, rabbit IgG from pooled rabbit serum was used instead of rabbit anti-rCRAMP.

Apoptosis was analysed in serial paraffin-embedded sections of draining inguinal lymph nodes of DA rats by staining with an antibody to cleaved caspase 3 (Cell Signaling, Beverly, USA). 

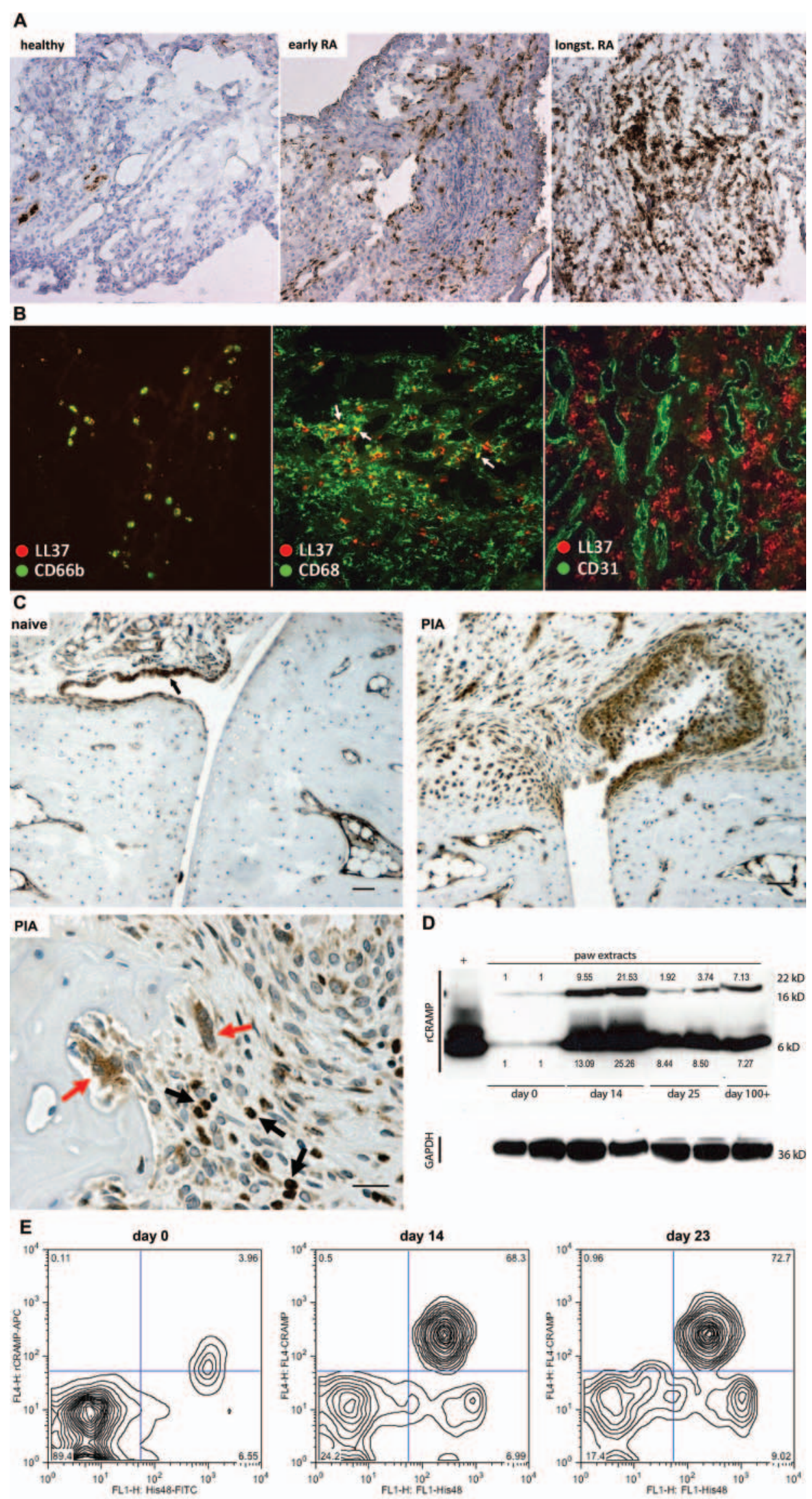

Figure 1 Continued. 
Statistical analyses

Comparisons of positive cells in flow cytometry and histology were made by analysis of variance tests. Where significant effects were found, subsequent comparisons were performed using Dunnett's test or Bonferroni corrections. Comparisons with premorbid values within groups (percentage positive cells in fluorescence-activated cell sorting from peripheral blood, PBMCs and neutrophil counts, serum IFN $\alpha$ levels) were tested using repeated-measures analysis of variance with Dunnett's test or Bonferroni correction. Levels of significance are labelled throughout the figures as follows: ${ }^{*} p<0.05,{ }^{* *} p<0.01,{ }^{* * *} p<0.001$.

\section{RESULTS}

Expression of cathelicidins is increased in joints during inflammatory arthritis

To determine whether LL-37 is locally overexpressed in the inflamed joints during RA at a protein level, we analysed expression in the synovial membranes of patients with early and longstanding RA by immunohistochemistry. In the healthy synovium LL-37 was expressed in only a few vessels, but we found clear upregulation of expression with the development of RA (figure 1A). In the arthritic synovium, LL-37 was mainly expressed in CD66b granulocytes (figure 1B) and in some CD68 macrophages (figure 1B), whereas CD31 local endothelial cells did not express LL-37 (figure 1B). The predictable, reliable and multiphase disease course of PIA provides an opportunity to analyse the expression of rCRAMP before onset and during different stages of arthritis (see online supplementary Figure S1a). In naïve rats, immunohistochemistry disclosed expression of rCRAMP in cells of the synovial lining and weak expression in some chondrocytes. With development of arthritis, rCRAMP was strongly expressed in cells of the inflammatory pannus tissue, mostly of macrophage, fibroblast and granulocyte morphology. Similar to the pattern of expression in humans, strongest expression could be detected in neutrophil granulocytes and intermediate expression in multinucleated osteoclasts resorbing the bone surface (figure 1C).

We then performed a comprehensive expression analysis in paws, lymphoid organs, peripheral blood and control organs in naïve (day 0) and pristane-injected rats before the onset of clinical symptoms (day 7), around onset (day 14), at the peak of the acute phase (day 23) and during the chronic phase of PIA (day $100+$ ). Expression analysis by immunoblot in paws showed overexpression of the proform and even stronger upregulation of the mature $7 \mathrm{kDa}$ rCRAMP peptide during arthritis, most pronounced at the very early phase of PIA (day 14) but sustained until the late, chronic stage (figure $1 \mathrm{D})$.
Flow cytometry analysis of SF samples obtained at different stages of PIA revealed that rCRAMP is expressed almost exclusively in cells recognised by the antibody His48 (denoted His48+ cells) (figure 1E) showing mostly granulocyte but partly also monocyte morphology in forward-scatter/side-scatter (FSC/SSC) plots. His48 is commonly used as a phenotypic marker for rat granulocytes. Interestingly, cells expressing His48medium cells stained most strongly positive for rCRAMP, whereas His $48^{\text {high }}$ cells, constituting monocytes and granulocytes (see online supplementary Figure S1b), were rCRAMP-. Apart from expression in His $48+$ cells, rCRAMP was induced after pristane injection in the SF in a significantly higher number of CD68 and $\gamma \delta \mathrm{T}$ cells. Expression in B and $\alpha \beta$ T cells was upregulated at very low levels (see online supplementary Table SI).

Similar to rCRAMP, albeit expressed in a lower percentage of cells, the number of defensin $\alpha 4$-expressing cells was significantly increased concomitant with the infiltration of His48+ cells into the joint around the onset of PIA and increased further in the acute phase. In contrast, defensin- $\beta 1$ was not expressed in SF cells (see online supplementary Figure S1c).

\section{Cathelicidin expression in secondary lymphoid organs and peripheral blood during PIA}

Also, in the spleen, upregulation of rCRAMP expression was seen with development of arthritis, although overexpression was less strong than in affected joints. Notably, the mature rCRAMP peptide rather than the proform was predominantly expressed in spleens already in naïve rats (probably accounting for the presence of resident neutrophils) and remained preponderant during arthritis (figure 2A). Again, rCRAMP was mainly expressed in His48+ splenic cells and became more common in $\gamma \delta \mathrm{T}$ cells during arthritis (see online supplementary Table SI). Similar to rCRAMP cells, also defensin- $\alpha 4$ and defensin- $\beta 1$ cells were more common in spleen during PIA (see online supplementary Figure S2a).

The upregulation of expression in the spleen was confirmed at transcriptional level by Affymetrix gene array, identifying AMPs among the most highly upregulated genes at days 10 and 20 after pristane injection (see online supplementary Table SII).

Likewise, western blots performed from whole peripheral blood showed a higher expression of rCRAMP after pristane injection. In this case, the proform was dominantly expressed, suggesting the presence of a higher percentage of non-activated neutrophils in the peripheral blood than in SF (figure 2B). After pristane injection, increase in the percentage of both His48 and rCRAMP-expressing cells was dramatic and occurred before onset of clinically discernible arthritis (figure 2C).

Figure 1 Articular expression of cathelicidins during arthritis. (A) Representative immunohistochemistry of LL-37 in synovial membranes of a healthy donor, a patient with early rheumatoid arthritis (RA) and a patient with longstanding RA. Whereas in the healthy donor, LL-37 expression is refined to a few cells in the vessels, expression is enhanced within the synovium during arthritis. (B) Double immunofluorescence staining of LL-37 and CD66b, CD68 or CD31, respectively, in a patient with early RA. Magnification $\times 10$. (C) Immunohistochemical analysis of rCRAMP expression in hind paws of Dark Agouti rats at day 0 and day 23 after pristane injection. In naïve animals rCRAMP is expressed in synoviocytes (especially in the synovial lining, black arrow), part of the chondrocytes and in osteoblast-like cells of the bone marrow. During pristane-induced arthritis (PIA), rCRAMP is also strongly expressed in inflammatory cells of the infiltrating pannus tissue. Magnification $\times 40$ (lower panel) shows strongest expression in cells with neutrophil morphology (black arrows) and intermediate expression in macrophage-like cells and multinucleated osteoclasts (red arrows). (D) Western blot analysis of rCRAMP expression in joints during PIA. Protein extracts prepared from rat hind paws at various time points after pristane injection (extracts from three rats pooled per lane) were separated by sodium dodecyl sulphate-polyacrylamide gel electrophoresis (SDS-PAGE) and probed with polyclonal rabbit anti-rCRAMP. Synthetic rCRAMP served as positive control $(+)$, glyceraldehyde-3-phosphate dehydrogenase (GAPDH) as loading control. For densitometric analysis ratios between rCRAMP (proform or mature peptide, relatively) and GAPDH bands were calculated and upregulation during PIA was compared with protein bands from naïve rats. The figure shows a representative result of three experiments. (E) Flow cytometry-based analysis of rCRAMP expression in synovial fluid during PIA. rCRAMP is almost exclusively expressed in infiltrating His48+ cells 
A
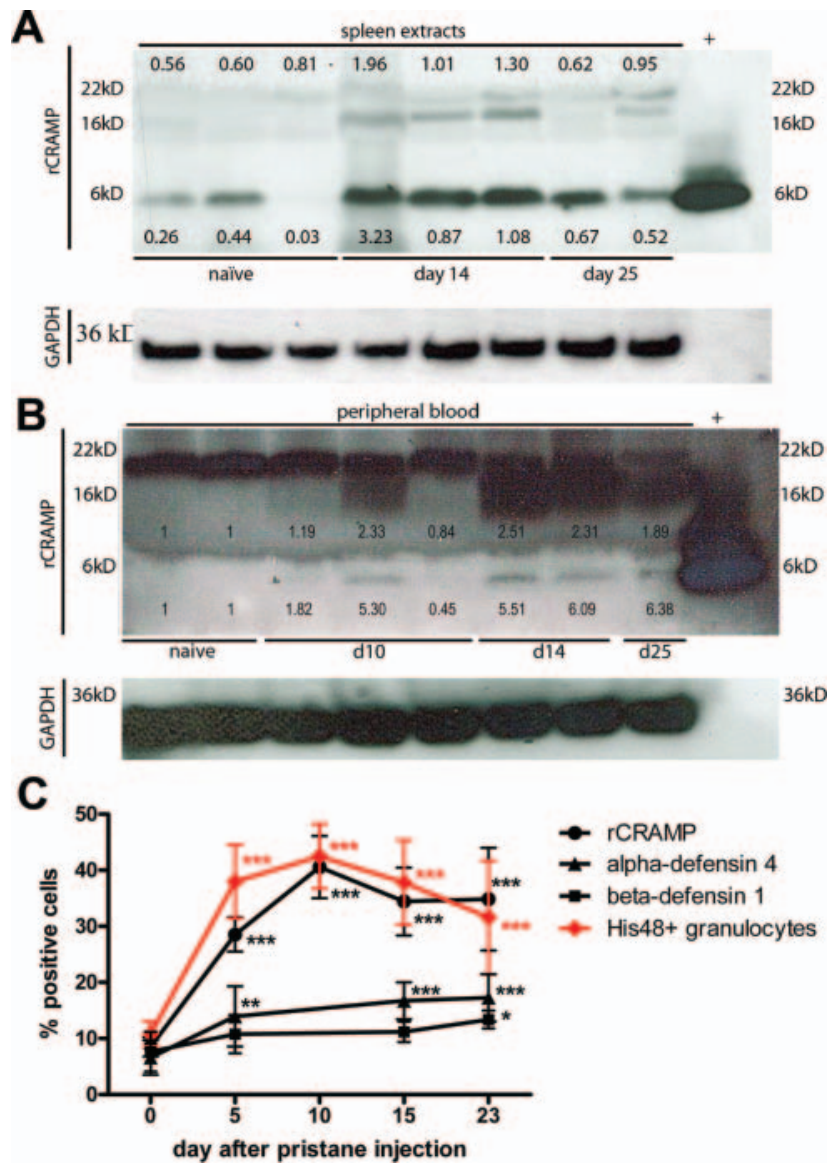

Figure 2 Expression of rCRAMP in spleen and peripheral blood during pristane-induced arthritis (PIA). (A) Western blot analysis of rCRAMP expression in spleens of naïve and pristane-injected Dark Agouti rats. Proteins (extracts of two rats pooled per lane) were separated by sodium dodecyl sulphate-polyacrylamide gel electrophoresis (SDS-PAGE) and probed with polyclonal anti-rCRAMP. Synthetic rCRAMP was run in parallel as positive control $(+)$,

glyceraldehyde-3-phosphate dehydrogenase (GAPDH) served as loading control. rCRAMP protein bands were analysed by densitometry, and expression levels compared with GAPDH bands are depicted. The figure shows a representative result of three experiments. (B) Western blot analysis of rCRAMP expression in whole peripheral blood, including erythrocytes. Proteins were separated by SDS-PAGE and probed with anti-rCRAMP (proteins from three rats pooled per lane). The intracellular proform of rCRAMP is expressed in all samples tested, whereas the mature rCRAMP peptide was only detected in pristane-injected animals. For densitometric analysis ratios between rCRAMP (proform or mature peptide, relatively) and GAPDH bands were calculated. Upregulation during PIA was compared with bands from naïve rats. The figure shows one of two independent experiments. (C) Time course of percentages of His $48+$ cells compared with number of antimicrobial peptide-expressing cells in peripheral blood of rats at various time points after pristane injection. Curves show mean $\pm S D$ of 6 . ${ }^{*} p<0.05$, ${ }^{* *} \mathrm{p}<0.01$, ${ }^{* *} \mathrm{p}<0.001$.

In contrast to spleen and peripheral blood, we did not observe accumulation of His48 cells or of rCRAMP-expressing cells in the pristane-draining inguinal lymph nodes. Also few if any defensin-expressing cells were detected (see online supplementary Figure S2b).

As a control for an organ not directly involved in the development of arthritis, we found no pristane-induced upregulation of rCRAMP in liver protein extracts during PIA (see online supplementary Figure S2c).

Phenotypic characterisation of cathelicidin-expressing cells in peripheral blood and SF

The vast majority of rCRAMP-positive cells in whole blood was bound by the His48 antibody and exhibited granulocyte morphology on FSC/SSC plots. On His48- cells, rCRAMP was only expressed on very small fractions of $B$ and $\alpha \beta$ T cells, but was expressed on higher numbers of $\gamma \delta \mathrm{T}$ cells after pristane injection (see online supplementary Table SI).

The unknown antigen recognised by His48 is believed to be exclusively expressed on rat granulocytes and cells of the erythroid lineage during their developmental stage in the bone marrow. Interestingly, rCRAMP cells bound by His48 also expressed low to intermediate levels of CD68. These His48+CD68 + cells exhibited both granulocyte and monocyte morphology on FSC/SSC and increased strongly in frequency after pristane priming (see online supplementary Table SI). His48+CD68+ cells showed substantially stronger expression of rCRAMP than His48+CD68cells in both naïve and pristane-primed animals (figure 3A). CD68 is a glycoprotein that binds to low-density lipoprotein and has been used as a marker for monocytes and macrophages. ${ }^{20} \mathrm{~A}$ population of granulocyte-like cells expressing macrophage markers has been described in Toxoplasma-challenged mice ${ }^{21}$ and in traumatised rat brain. ${ }^{22}$ Similarly, expression of these markers appears to be also less exclusive in the peripheral blood of DA rats. However, analysis by fluorescence-activated cell sorting and immunohistochemistry showed that rCRAMP can be expressed in cells with both granulocyte and monocyte morphology (see online supplementary Figure S3).

Importantly, rCRAMP expression was higher in activated than in resting His48+ cells in blood and SF, as determined by expression of CD62L (figure 3B,C). CD62L (L-selectin) is a cell adhesion molecule found on leukocytes that mediates the initial rolling on the endothelium. ${ }^{23}$ On neutrophils, CD62L is downregulated after activation by shedding. Although the number of CD62L + cells was decreased after pristane injection in both blood and SF, a substantial number of His48+ cells in blood were still CD62L+ and therefore in a resting state, whereas the vast majority of cells at the site of inflammation, in the SF, were activated (CD62L-).

Cathelicidins are highly expressed in neutrophil granules, but cell surface LL-37 has been described on activated neutrophils of patients with SLE. ${ }^{3}$ We tested flow cytometry staining of rCRAMP also on non-permeabilised cells isolated from peripheral blood of naive and pristane-primed rats but could not detect rCRAMP expression on the surface (see online supplementary Figure S4).

\section{Low-density neutrophils accumulate in the blood after pristane priming}

We found increased absolute numbers of neutrophils and PBMCs in peripheral blood after pristane priming (see online supplementary Figure S5a). Interestingly, we found slightly higher expression of rCRAMP and its proform in PBMCs from pristane-primed rats than from naïve rats on western blot (figure 4A). This came as a surprise since in whole peripheral blood rCRAMP expression had been largely restricted to His48+ cells and granulocytes normally cannot be found in the same layer as PBMCs after density gradient centrifugation. Flow cytometry showed an increased percentage of His48+ cells copurifying with PBMCs during density gradient centrifugation in pristane-primed rats (figure 4B), possibly accounting for the upregulated expression of rCRAMP in PBMCs. 
Downloaded from http://ard.bmj.com/ on October 26, 2016 - Published by group.bmj.com

\section{Basic and translational research}
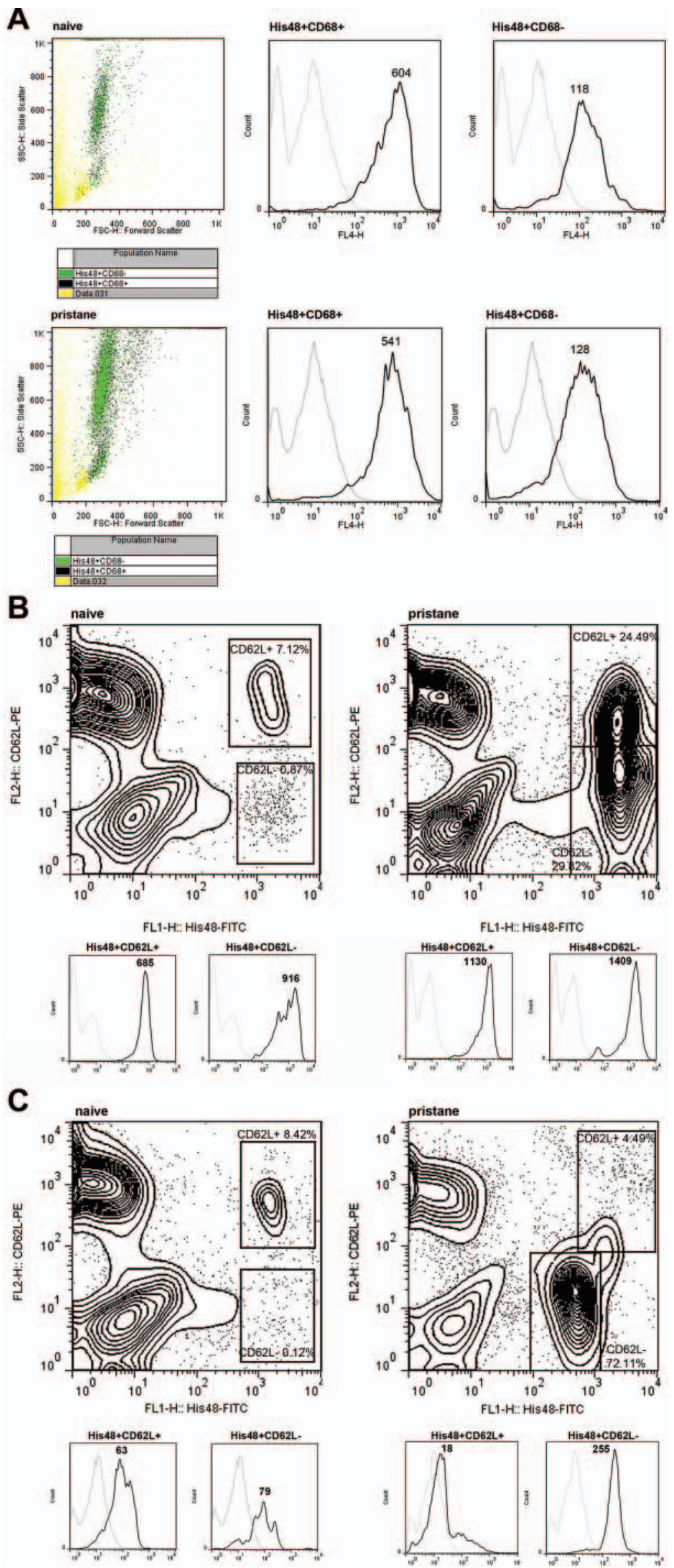

Figure 3 Continued. 

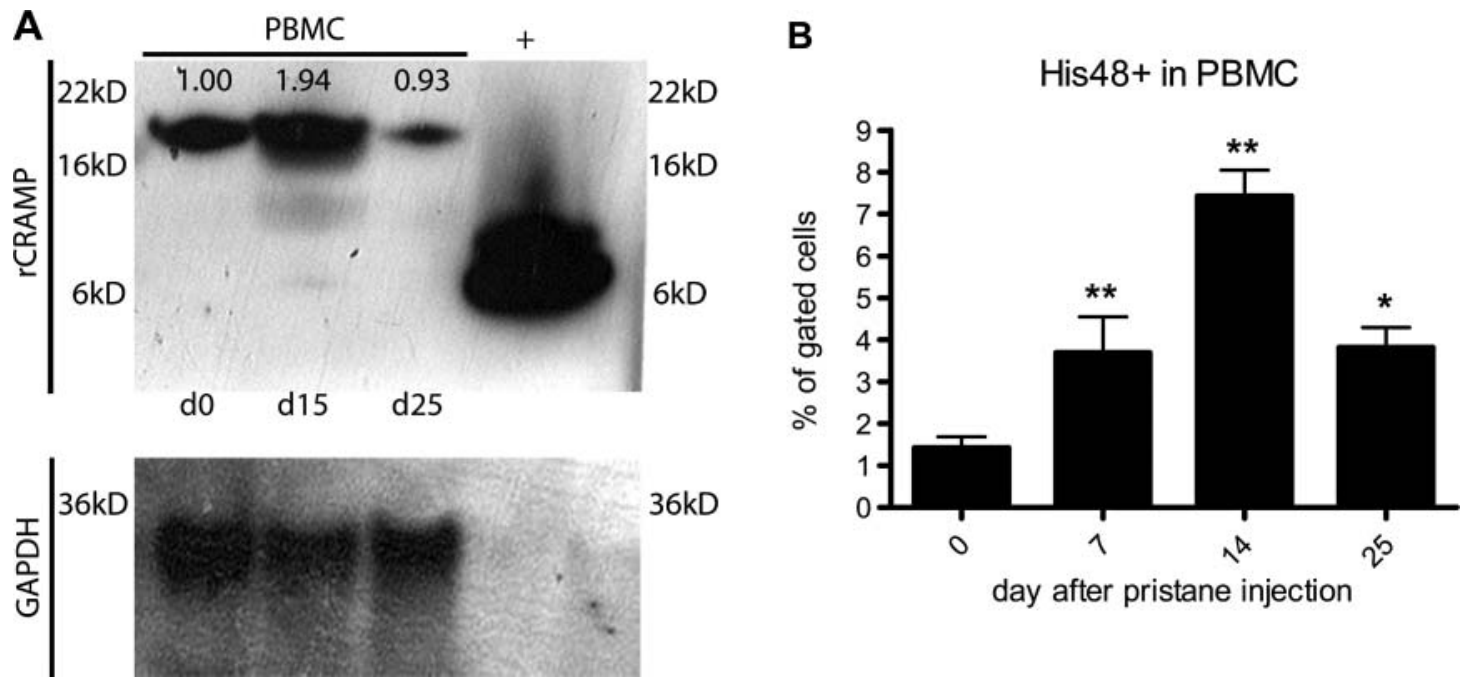

Figure 4 Pristane triggers accumulation of low-density blood neutrophils and expression of antimicrobial peptides (AMPs). (A) Western blot analysis of rCRAMP expression in peripheral blood mononuclear cells (PBMCs) isolated by conventional density gradient centrifugation. Lanes show pools of proteins extracted from four animals each, separated by sodium dodecyl sulphate-polyacrylamide gel electrophoresis and stained with rabbit anti-rCRAMP. Glyceraldehyde-3-phosphate dehydrogenase (GAPDH) was used as loading control. Densitometric analysis shows slight upregulation of rCRAMP expression in PBMCs isolated from rats 15 days after pristane injection (B). Percentage of His48+ cells in the PBMC fraction after density gradient centrifugation performed on blood of rats before and at various time points after pristane injection. Each bar shows mean $\pm S D$ from five animals. ${ }^{*} p<0.05,{ }^{* *} p<0.01$.

rCRAMP expression in the PBMC layer was mostly apparent in His48+ cells that showed granulocyte morphology on FSC/ SSC (see online supplementary Figure S5b).

Autoantibodies and increased apoptosis levels in the draining lymph nodes coincide with higher levels of IFNo at the onset of PIA

In SLE, immunogenic self-DNA-AMP complexes lead to the development of antibodies against both DNA and AMPs. ${ }^{3}$ To test if similar processes also occur in PIA, we tested serum samples of rats at days $0,6,14,25$ and $100+$ after pristane injection for the presence of $\operatorname{IgG}$ and IgM autoAbs against rCRAMP. IgG autoAbs were found from day 14 after pristane injection and levels were maintained during the course of PIA, peaking in strength at the acute phase of the disease on day 25 (figure 5A). Specificity of the bands on western blot was confirmed by partial inhibition of the signal by preincubation of the membrane with a polyclonal rabbit IgG anti-rCRAMP antibody or by preincubation of the serum samples with rCRAMP (see online supplementary Figure S6).

We also found IgM autoantibodies to rCRAMP in the serum samples of rats, particularly at early time points (figure $5 B$ ). In contrast, we could not detect anti-rCRAMP autoAbs in a pool of serum samples from rats that had been injected with arthritogenic cells (not shown), indicating that development of autoantibodies is dependent on early pathogenic events directly caused by pristane.

Pristane has been shown to induce cell death when applied in vitro or upon intraperitoneal injection in mice. ${ }^{24}$ After intradermal injection at the base of the tail, the arthritogenic oil hexadecane disseminates mainly to the inguinal lymph nodes. ${ }^{26}$ Since the dynamics of hexadecane-induced arthritis and PIA are closely related, a similar dissemination scheme is expected for pristane. An increased frequency of apoptotic cells determined by immunohistochemical staining of cleaved caspase 3 was apparent in the pristane-draining inguinal lymph nodes $24 \mathrm{~h}$ after pristane injection, peaked at $72 \mathrm{~h}$ and then gradually returned to levels detected in naïve animals (figure 5C). Apoptosis was particularly visible in the marginal sinus of the lymph nodes (see online supplementary Figure S8).

Under conditions of insufficient clearing, increased cell death may be associated with exposure of cellular and nuclear contents to the immune system, causing activation of endosomal TLRs and production of type I IFN. In our system, the occurrence of autoantibodies and high levels of cell death in lymph nodes coincided with increased serum levels of IFN $\alpha$ during early PIA (figure 5D). Interestingly, IFN $\alpha$ serum levels were not increased in the transfer model of PIA. This suggests that the production of IFN $\alpha$ is related to mechanisms that are directly caused by exposure to pristane.

\section{DISCUSSION}

Upon priming by bacterial products and cytokines or chemokines, neutrophils are mobilised to the site of infection or inflammation, where they release their granule contents and form neutrophil extracellular traps. ${ }^{27}$

An important group of molecules released by activated neutrophils in inflamed tissues are the AMPs. The two most

Figure 3 Characterisation of rCRAMP-expressing cells in blood and synovial fluid. (A) His48+CD68+ cells express the highest amounts of rCRAMP. Representative flow cytometry plots from peripheral blood drawn from a naïve rat and a rat 21 days after pristane injection and stained with CD68-fluorescein isothiocyanate (FITC), His48-phycoerythrin (PE) and rCRAMP-Alexa 647. Cells expressing both His48 and CD68 at high levels exhibit both neutrophil and monocyte morphology on forward-scatter/side-scatter (FSC/SSC) plots and express higher GeoMeans of rCRAMP mean fluorescence intensity than His48hi CD68- cells. (B and C) His48+CD62L- cells increase in percentage in peripheral blood and synovial fluid (SF) after pristane priming. Representative flow cytometry plots from peripheral blood (B) and SF (C) of a naïve and pristane-primed (day 21) rat. Expression of rCRAMP is markedly higher in activated CD62L- cells than with resting CD62L-cells. 
A

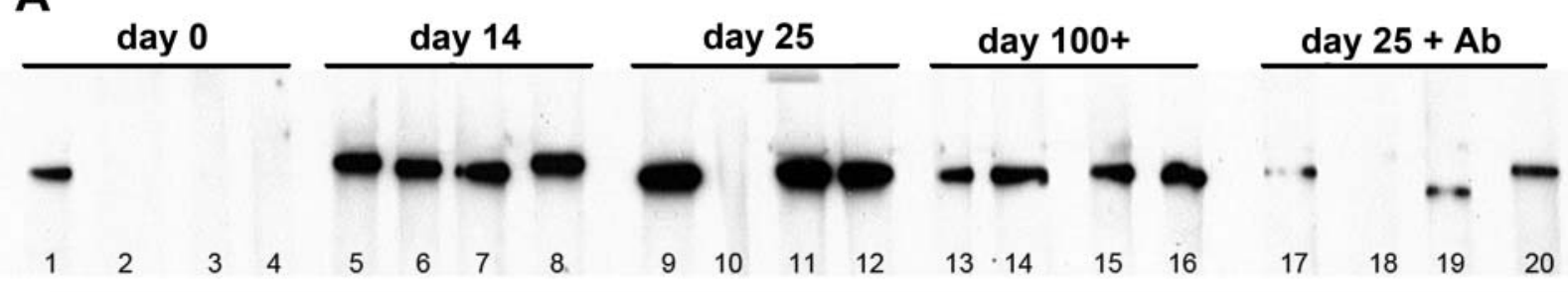

B
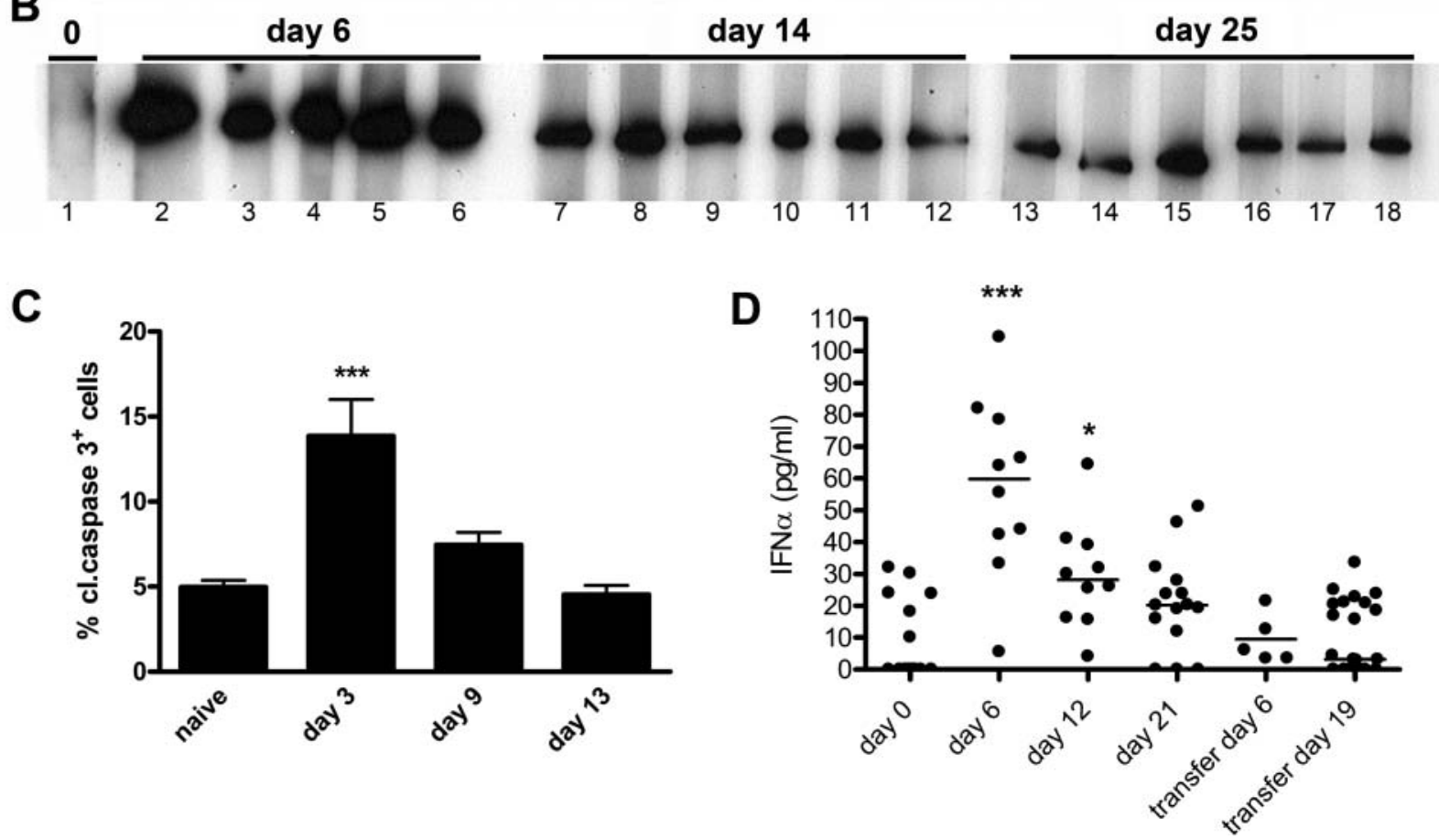

Figure 5 Autoantibodies to rCRAMP are found together with increased apoptosis and raised serum levels of interferon (IFN $\alpha$ ) in pristane-injected rats. Western blot analysis of anti-rCRAMP $\lg G(A)$ and $\operatorname{lgM}(B)$ autoantibodies in serum samples of rats at various time points after injection of pristane, using blotted rCRAMP peptide. Some lanes were preincubated with a polyclonal rabbit anti-rCRAMP antibody to control for specificity of the observed bands. Each lane represents a strip incubated with a serum sample of one individual rat, except for IgM day 0, where serum samples of five animals have been pooled. (C) Quantification of apoptotic cells in lymph nodes from naive and pristane-injected rats. Bars show mean \pm SEM from pooled lymph nodes of seven individual animals for each time point. (D) Determination of IFN $\alpha$ serum levels in rats at various time points after injection of pristane or transfer of arthritogenic lymph node cells, respectively. ${ }^{* *} p<0.001$.

thoroughly characterised families of AMPs are the defensins and the cathelicidins. ${ }^{28}$ Defensins are cationic amphipathic peptides with a triple-stranded antiparallel $\beta$-sheet structure stabilised by three characteristic disulphide bonds and can be further subdivided into $\alpha$-, $\beta$ - and $\theta$-defensins, based on molecular weight and the pattern of disulphide bonding. In humans, rats and most other mammals, $\alpha$-defensins $1-4$ are mainly found in the secretory granules of neutrophils. In contrast, $\beta$-defensins are not expressed in neutrophils, but by most epithelial cells, and their expression is often stimulated by proinflammatory stimuli and infection. In the rat, the $\alpha$-defensins RNP $1-4$ and the $\beta$-defensins RBD 1-2 are known. ${ }^{29-31}$

Similar to defensins, cathelicidins are synthesised and stored as inactive precursors in neutrophil granules and are proteolytically maturated before they are released as active AMPs. ${ }^{32} 33$ In humans there is only one known cathelicidin precursor protein, hCAP18, which yields the 37 amino acid long mature peptide LL-37. ${ }^{34}{ }^{35}$ Rats also have only one cathelicidin precursor, which is processed to produce the mature 43-residue peptide rCRAMP. ${ }^{19}$

Neutrophils are the most abundant immune cells in the SF of patients with RA but may also be found at the pannus/cartilage interface in synovial tissue, ${ }^{36} 37$ especially in the early stages of RA. Animal models of inflammatory arthritis show an early entrance of neutrophils to the arthritic joint that is associated with development of oedema and joint swelling. ${ }^{38-41}$ The very strong expression of rCRAMP that we observed in the early stages of PIA is therefore presumably transported by the high number of neutrophils entering the pannus from the SF. At later stages, rCRAMP-expressing cells of the monocyte/macrophage lineage might also contribute significantly.

The reason why hydrocarbon oils induce autoimmune inflammation in mammals continues to be debated. While pristane has been used for inducing autoimmune lupus and arthritis in mice and rats for decades, ${ }^{16} 4243$ and is associated with these diseases upon environmental exposure in humans, ${ }^{44} 45$ its mechanism of action is still elusive. Major histocompatibility complex class II-restricted T cells are crucially involved in the development of PIA in rats, ${ }^{46}$ but other mechanisms such as induction of cell death and direct induction of inflammatory cytokines by pristane might also contribute to its pathogenesis. ${ }^{25}$

Another potential pathogenic mechanism is induction of systemic inflammation driven by neutrophils. In SLE, increased occurrence in blood of low-density neutrophils copurifying 
with PBMCs during gradient centrifugation has been described. Their presence has been attributed to premature release from the bone marrow owing to recruitment of mature neutrophils to sites of inflammation, or owing to accelerated mature neutrophil turnover. ${ }^{47} 48$ We detected low buoyant-density neutrophils also in pristane-injected rats, suggesting that similar mechanisms might be at work during inflammatory arthritis.

The direct effects of pristane can be distinguished well from its secondary effects, which are driven by articular inflammation, by comparing disease parameters in PIA with those in $\mathrm{T}$ cell-transferred PIA. Although the transfer is strictly dependent on pristane priming of the donor cells, the acceptor rats develop an arthritis that is very similar to PIA without having been in direct contact with the oil. We found upregulation of rCRAMP expression and accumulation of neutrophils in both PIA and the PIA transfer model, but with different kinetics (see online supplementary Figure S7). In contrast, induction of serum IFN $\alpha$ and development of autoantibodies are restricted to PIA, indicating that they are caused by direct effects of pristane operating independently of T cell activation-for example, related to a B cell response against stressed and dying cells.

In summary, we performed a comprehensive expression analysis in joints, lymphoid organs and blood and detected strong overexpression of cathelicidins during arthritis. In the joint, cathelicidins were mainly expressed in cells of the inflammatory pannus, mostly those of monocytic, fibroblastoid and granulocytic morphology. A phenotypic characterisation of rCRAMP-expressing cells in peripheral blood and SF showed that rCRAMP expression is highest in activated CD62L- cells coexpressing granulocyte and monocyte markers and exhibiting granulocyte or monocyte morphology. Furthermore, IgG and IgM autoantibodies to rCRAMP were triggered by pristane and coincided with increased levels of cell death and increased serum levels of IFN $\alpha$.

The possibility that global and local overexpression of AMPs directly influences development and/or maintenance of arthritis will be examined in future studies. Local effects might be elicited by excessive release of mature AMPs that exert immunemodulating and chemotactic effects. Formation of complexes between cationic AMPs and locally released nucleic acids may alert the immune system by the activation of TLR7 and/or TLR9 expressed in the arthritic joints. Systemic effects might be fuelled by the development of autoantibodies and immune complexes in a way that has been described in $\mathrm{SLE}^{2}{ }^{3}$ or by influencing $\mathrm{T}$ cell polarisation.

Acknowledgements The authors thank Monica Lindh for technical help in establishing the western blot analysis and peptide extraction protocols, Carl-Walter Steiner for help with fluorescence activated cell sorting of rCRAMP-positive cells, Marianne Engström for technical help with immunohistochemistry of human samples and Anita Fischer, Ulrika Norin and Jonatan Tuncel for providing rat samples.

Contributors $\mathrm{MHH}, \mathrm{MH}, \mathrm{AIC}, \mathrm{BA}$ and $\mathrm{RH}$ were involved in conception of the study. $\mathrm{MHH}, \mathrm{LB}, \mathrm{PN}, \mathrm{HB}$ and $\mathrm{BN}$ were involved in performing experiments. All authors approved the final version to be published. BA and $\mathrm{RH}$ contributed equally to this work.

Funding The research was funded by the Austrian Science Fund FWF (roject J3102-B13), the Swedish Research Council, the Swedish Strategic Foundation (SSF), the Lars Hiertas minne foundation, the EU project Masterswitch (HEALTH-F2-2008-223404) and by the IMl program BTCure.

Competing interests None.

Ethics approval Local ethical committee, Sweden.

Provenance and peer review Not commissioned; externally peer reviewed.

Correction notice This article has been corrected since it was published Online First. Correspondence and author affiliations have been corrected. Occurrences of "His48 cells", "CD62 cells" and "pristine" have been changed to "His48+ cells", "CD62 + cells" and "pristane" respectively.

\section{REFERENCES}

1. Ganguly D, Chamilos G, Lande R, et al. Self-RNA-antimicrobial peptide complexes activate human dendritic cells through TLR7 and TLR8. J Exp Med 2009;206:1983-94.

2. Garcia-Romo GS, Caielli S, Vega B, et al. Netting neutrophils are major inducers of type IFN production in pediatric systemic lupus erythematosus. Sci Trans/ Med 2011;3:73ra20.

3. Lande R, Ganguly D, Facchinetti V, et al. Neutrophils activate plasmacytoid dendritic cells by releasing self-DNA-peptide complexes in systemic lupus erythematosus. Sci Transl Med 2011;3:73ra19.

4. Lande R, Gregorio J, Facchinetti V, et al. Plasmacytoid dendritic cells sense self-DNA coupled with antimicrobial peptide. Nature 2007;449:564-9.

5. Nijnik A, Hancock R. Host defence peptides: antimicrobial and immunomodulatory activity and potential applications for tackling antibiotic-resistant infections. Emerg Health Threats J 2009;2:e1.

6. De Y, Chen 0, Schmidt AP, et al. LL-37, the neutrophil granule- and epithelial cell-derived cathelicidin, utilizes formyl peptide receptor-like 1 (FPRL1) as a receptor to chemoattract human peripheral blood neutrophils, monocytes and T cells. J Exp Med 2000;192:1069-74

7. Mookherjee N, Brown KL, Bowdish DM, et al. Modulation of the TLR-mediated inflammatory response by the endogenous human host defense peptide LL-37. J Immunol 2006;176:2455-64

8. Morizane S, Yamasaki K, Muhleisen B, et al. Cathelicidin antimicrobial peptide LL-37 in psoriasis enables keratinocyte reactivity against TLR9 ligands. J Invest Dermatol 2012;132:135-43.

9. Baechler EC, Batliwalla FM, Karypis G, et al. Interferon-inducible gene expression signature in peripheral blood cells of patients with severe lupus. Proc Natl Acad Sci U S A 2003;100:2610-15.

10. Bennett L, Palucka AK, Arce E, et al. Interferon and granulopoiesis signatures in systemic lupus erythematosus blood. J Exp Med 2003;197:711-23.

11. van der Pouw Kraan TC, Wijbrandts CA, van Baarsen LG, et al. Rheumatoid arthritis subtypes identified by genomic profiling of peripheral blood cells: assignment of a type I interferon signature in a subpopulation of patients. Ann Rheum Dis 2007;66:1008-14.

12. Frohm M, Agerberth B, Ahangari G, et al. The expression of the gene coding for the antibacterial peptide LL-37 is induced in human keratinocytes during inflammatory disorders. J Biol Chem 1997;272:15258-63.

13. Sun CL, Zhang FZ, Li P, et al. LL-37 expression in the skin in systemic lupus erythematosus. Lupus 2011;20:904-11.

14. Paulsen F, Pufe T, Conradi L, et al. Antimicrobial peptides are expressed and produced in healthy and inflamed human synovial membranes. J Pathol 2002;198:369-77

15. Matsumoto $\mathbf{T}$, Kaneko $\mathrm{T}$, Seto $\mathrm{M}$, et al. The membrane proteinase 3 expression on neutrophils was downregulated after treatment with infliximab in patients with rheumatoid arthritis. Clin Appl Thromb Hemost 2008;14:186-92.

16. Vingsbo C, Sahlstrand P, Brun JG, et al. Pristane-induced arthritis in rats: a new model for rheumatoid arthritis with a chronic disease course influenced by both major histocompatibility complex and non-major histocompatibility complex genes. Am J Pathol 1996;149:1675-83.

17. Hoffmann MH, Tuncel J, Skriner K, et al. The rheumatoid arthritis-associated autoantigen hnRNP-A2 (RA33) is a major stimulator of autoimmunity in rats with pristane-induced arthritis. J Immunol 2007;179:7568-76.

18. Russo-Carbolante EMS, Azzolini AECS, Plizello ACM, et al. Comparative study of four isolation procedures to obtain rat neutrophils. Comp Clin Pathol 2002;11:71-6.

19. Termen S, Tollin M, Olsson B, et al. Phylogeny, processing and expression of the rat cathelicidin rCRAMP: a model for innate antimicrobial peptides. Cell Mol Life Sci 2003;60:536-49.

20. Holness CL, Simmons DL. Molecular cloning of CD68, a human macrophage marker related to lysosomal glycoproteins. Blood 1993;81:1607-13.

21. Mordue DG, Sibley LD. A novel population of Gr-1+-activated macrophages induced during acute toxoplasmosis. J Leukoc Biol 2003;74:1015-25.

22. Matsumoto H, Kumon $Y$, Watanabe $H$, et al. Antibodies to CD11b, CD68 and lectin label neutrophils rather than microglia in traumatic and ischemic brain lesions. J Neurosci Res 2007;85:994-1009.

23. Gearing AJ, Newman W. Circulating adhesion molecules in disease. Immunol Today 1993;14:506-12.

24. Calvani N, Caricchio R, Tucci $\mathrm{M}$, et al. Induction of apoptosis by the hydrocarbon oil pristane: implications for pristane-induced lupus. J Immunol 2005;175:4777-82

25. Herman S, Kny A, Schorn C, et al. Cell death and cytokine production induced by autoimmunogenic hydrocarbon oils. Autoimmunity 2012; Epub ahead of print.

26. Kleinau S, Dencker L, Klareskog L. Oil-induced arthritis in DA rats: tissue distribution of arthritogenic 14C-labelled hexadecane. Int J Immunopharmacol 1995;17:393-401

27. Fuchs TA, Abed U, Goosmann C, et al. Novel cell death program leads to neutrophil extracellular traps. J Cell Biol 2007;176:231-41.

28. Cederlund A, Gudmundsson GH, Agerberth B. Antimicrobial peptides important in innate immunity. FEBS J 2011;278:3942-51. 
29. Yount NY, Wang MS, Yuan J, et al. Rat neutrophil defensins. Precursor structures and expression during neutrophilic myelopoiesis. J Immunol 1995;155:4476-84.

30. Jia HP, Mills JN, Barahmand-Pour F, et al. Molecular cloning and characterization of rat genes encoding homologues of human beta-defensins. Infect Immun 1999;67:4827-33.

31. Eisenhauer PB, Harwig SS, Szklarek D, et al. Purification and antimicrobial properties of three defensins from rat neutrophils. Infect Immun 1989;57:2021-7.

32. Sorensen $\mathbf{0 E}$, Follin $\mathrm{P}$, Johnsen AH, et al. Human cathelicidin, hCAP-18, is processed to the antimicrobial peptide LL-37 by extracellular cleavage with proteinase 3. Blood 2001;97:3951-9.

33. Zanetti M, Gennaro R, Romeo D. Cathelicidins: a novel protein family with a common proregion and a variable C-terminal antimicrobial domain. FEBS Lett 1995;374:1-5

34. Turner J, Cho Y, Dinh NN, et al. Activities of LL-37, a cathelin-associated antimicrobial peptide of human neutrophils. Antimicrob Agents Chemother 1998:42:2206-14.

35. Johansson J, Gudmundsson GH, Rottenberg ME, et al. Conformation-dependent antibacterial activity of the naturally occurring human peptide LL-37. J Biol Chem 1998:273:3718-24

36. Mohr W, Kohler G, Wessinghage D. Polymorphonuclear granulocytes in rheumatic tissue destruction. II. Demonstration of PMNs in rheumatoid nodules by electron microscopy. Rheumatol Int 1981;1:21-8.

37. Wright HL, Moots RJ, Bucknall RC, et al. Neutrophil function in inflammation and inflammatory diseases. Rheumatology (Oxford) 2010;49:1618-31.

38. Chou RC, Kim ND, Sadik CD, et al. Lipid-cytokine-chemokine cascade drives neutrophil recruitment in a murine model of inflammatory arthritis. Immunity 2010:33:266-78.

39. Holmdahl R, Jansson L, Larsson A, et al. Arthritis in DBA/1 mice induced with passively transferred type II collagen immune serum. Immunohistopathology and serum levels of anti-type II collagen auto-antibodies. Scand J Immunol 1990;31:147-57.

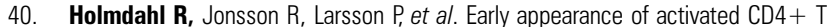
lymphocytes and class II antigen-expressing cells in joints of DBA/1 mice immunized with type II collagen. Lab Invest 1988;58:53-60.

41. Wipke BT, Allen PM. Essential role of neutrophils in the initiation and progression of a murine model of rheumatoid arthritis. J Immunol 2001;167:1601-8.

42. Satoh M, Reeves WH. Induction of lupus-associated autoantibodies in BALB/C mice by intraperitoneal injection of pristane. J Exp Med 1994;180:2341-6.

43. Wooley PH, Seibold JR, Whalen JD, et al. Pristane-induced arthritis. The immunologic and genetic features of an experimental murine model of autoimmune disease. Arthritis Rheum 1989:32:1022-30.

44. Dahlgren J, Takhar H, Anderson-Mahoney P. Cluster of systemic lupus erythematosus (SLE) associated with an oil field waste site: a cross sectional study. Environ Health 2007;6:8

45. Sverdrup B, Kallberg H, Bengtsson C, et al. Association between occupational exposure to mineral oil and rheumatoid arthritis: results from the Swedish EIRA case-control study. Arthritis Res Ther 2005; 7:R1296-303.

46. Holmberg J, Tuncel J, Yamada $\mathrm{H}$, et al. Pristane, a non-antigenic adjuvant, induces MHC class II-restricted, arthritogenic T cells in the rat. J Immunol 2006:176:1172-9.

47. Doreau A, Belot A, Bastid J, et al. Interleukin 17 acts in synergy with B cellactivating factor to influence $B$ cell biology and the pathophysiology of systemic lupus erythematosus. Nat Immunol 2009;10:778-85.

48. Lit LC, Wong CK, Tam LS, et al. Raised plasma concentration and ex vivo production of inflammatory chemokines in patients with systemic lupus erythematosus. Ann Rheum Dis 2006;65:209-15. 


\section{The cathelicidins LL-37 and rCRAMP are associated with pathogenic events of arthritis in humans and rats}

Markus H Hoffmann, Heiko Bruns, Liselotte Bäckdahl, Petra Neregård, Birgit Niederreiter, Martin Herrmann, Anca Irinel Catrina, Birgitta Agerberth and Rikard Holmdahl

Ann Rheum Dis 2013 72: 1239-1248 originally published online November 21, 2012

doi: 10.1136/annrheumdis-2012-202218

Updated information and services can be found at:

http://ard.bmj.com/content/72/7/1239

These include:

Supplementary Material

References

Email alerting service
Supplementary material can be found at:

http://ard.bmj.com/content/suppl/2012/11/20/annrheumdis-2012-2022 18.DC1.html

This article cites 47 articles, 27 of which you can access for free at: http://ard.bmj.com/content/72/7/1239\#BIBL

Receive free email alerts when new articles cite this article. Sign up in the box at the top right corner of the online article.

Topic
Collections

Articles on similar topics can be found in the following collections

Degenerative joint disease (4621)

Musculoskeletal syndromes (4930)

Immunology (including allergy) (5117)

Connective tissue disease (4234)

Systemic lupus erythematosus (566)

Rheumatoid arthritis (3245)

Inflammation (1244)

\section{Notes}

To request permissions go to:

http://group.bmj.com/group/rights-licensing/permissions

To order reprints go to:

http://journals.bmj.com/cgi/reprintform

To subscribe to BMJ go to:

http://group.bmj.com/subscribe/ 